\title{
OFERTA DE SÓDIO EM REFEIÇÕES DE UNIDADES DE ALIMENTAÇÃO E NUTRIÇÃO ESCOLAR
}

\author{
SODIUM OFFER IN MEALS IN A SCHOOL FOOD SERVICE
}

\author{
Isabela Cicaroni Ottoni ${ }^{1}$ \\ Mônica Glória Neumann Spinelli²
}

Resumo: Inúmeras evidências relacionam o excesso de sal consumido pela população com o aumento de doenças crônicas não transmissíveis. Dados da POF 2002-2003 indicam que o consumo de sódio da população brasileira é quase duas vezes maior que o recomendado. Esse estudo teve como objetivo avaliar a quantidade de sódio extrínseco utilizado em preparações de uma unidade de alimentação e nutrição terceirizada de uma escola privada de São Paulo, que atende, aproximadamente, 60 clientes. A oferta total dos alimentos servidos foi obtida pesandose todas as preparações e subtraindo-se as sobras. Os restos não foram analisados neste trabalho. Para calcular a quantidade de sódio de cada preparação, foi pesada a quantidade de sal, de tempero industrializado para arroz e outros temperos ricos em sódio antes de serem utilizados no preparo. Foi observada uma oferta média per capita de 1099,5mg de sódio, valor maior que o recomendado pelo PAT. A oferta de sódio nessa unidade estudada é alto. É importante que se faça uma adequação como medida de prevenção de risco à saúde e com caráter educativo, já que se trata de refeição de unidade escolar. Os serviços de alimentação têm, por dever, proteger a saúde de seus clientes e colaboradores, e, portanto, devem ser mais criteriosos com a quantidade de componentes prejudiciais à saúde utilizados em suas preparações, além de padronizarem as receitas, indicando as quantidades de sal a serem utilizadas.

Palavras-chave: consumo de alimentos; sódio na dieta; serviços de alimentação; escola.

Abstract: There is extensive evidence relating the increase of chronic diseases with the extreme amount of salt consumed by the population. Data from 2002-2003 indicates that the Brazilian population's sodium intake is almost two times higher than recommended. This study aimed to evaluate the amount of extrinsic sodium used in preparations at a private school food service in the city of São Paulo, which serves daily about 60 customers. The total food supply served was obtained by weighing all preparations and subtracting leftovers. The leftovers were not analyzed in this work. In order to calculate the sodium amount used in each preparation, the salt, industrialized rice seasoning, and other sodium rich seasonings were weighted before being used in the preparation. The per capita sodium consumption of $1099.5 \mathrm{mg}$ was observed, which is higher than recommended by PAT. The sodium consumption at this unit can be considered high. Every food service unit should be responsible for protecting their costumers and co-workers' health by being more concerned and cautious about the amount of components used in preparation that can be harmful to health. They should also standardize their recipes, indicating the amount of salt that should be used

Keywords: food consumption; dietary sodium; food service; school.

\footnotetext{
1 Graduada em Nutrição - Universidade Presbiteriana Mackenzie - UPM. E-mail: isabela_ottoni@hotmail.com. 2 Doutora em Saúde Pública - Universidade de São Paulo - USP e Professora Adjunta I da UPM. E-mail: monica.spinelli@mackenzie.br.
} 


\section{INTRODUÇÃO}

Frente às evidências que relacionam excesso do consumo de gorduras, açúcares e sódio ao aumento do desenvolvimento de doenças crônicas não transmissíveis (DCNT), são inevitáveis orientações de caráter restritivo à ingestão desses grupos alimentares (BRASIL, 2005).

O sódio é um mineral essencial para a regulação dos fluidos intra e extracelular, atuando na manutenção da pressão sanguínea. É o principal componente do sal de cozinha (cloreto de sódio) que contém $40 \%$ de sódio em sua composição. Evidências atuais sugerem que o consumo menor que $1,7 \mathrm{~g}$ de sódio ( $5 \mathrm{~g}$ de cloreto de sódio por dia) pode contribuir para a redução da pressão arterial. A maior parte dos indivíduos, mesmo as crianças, consomem níveis desse mineral acima de suas necessidades. Além de fonte de sódio, o sal de cozinha é a fonte principal de iodo na alimentação do brasileiro (BRASIL, 2005).

Entretanto, o consumo excessivo de sal, maior que $6 \mathrm{~g}$ por dia, está relacionado ao aparecimento de diversas doenças, como a osteoporose e doenças circulatórias, como hipertensão arterial e doenças cardiovasculares (BRASIL apud MCBEAN et al., 1994). Estimase que a Hipertensão Arterial ocorra com incidência de $20 \%$ na população total brasileira (BRASIL, 2005).

Embora exista o consenso de que o consumo excessivo de alimentos com alto teor de sódio possa acarretar doenças renais, cardíacas e elevar a pressão arterial, quadros que melhoram com a diminuição do sódio (WORLD HEALTH ORGANIZATION, 2007), grande parcela da população brasileira consome sal em excesso (SALAS; SPINELLI, 2007). Estimase que o consumo médio diário da população brasileira seja de 9,6g de sal por pessoa, ou seja, o dobro do recomendado pelo Ministério da Saúde e pela Organização Mundial da Saúde (5g por dia ou $2 \mathrm{~g}$ de sódio) (BRASIL, 2008; WORLD HEALTH ORGANIZATION, 2007).

Segundo o Instituto Brasileiro de Geografia e Estatística (2001), mais que 70\% da população consome quantidades superiores ao valor máximo de ingestão tolerável para o sódio, sendo que a proporção de indivíduos com ingestão de sódio acima do nível seguro de ingestão foi mais elevada nas áreas urbanas, quando comparada às áreas rurais. Entre os gêneros, os homens apresentam uma maior inadequação no consumo desse nutriente que as mulheres, sendo a ingestão de sódio acima do nível seguro de ingestão, de $89 \%$ entre os homens e de $70 \%$ entre as mulheres, para a faixa etária de 19 a 59 anos, e de $80 \%$ e $62 \%$, respectivamente, para homens e mulheres com 60 anos ou mais de idade. Essa inadequação também é encontrada em adolescentes, entre os quais $70 \%$ desses tiveram ingestão superior ao valor máximo de ingestão tolerável.

Atualmente, cerca de 18 milhões de mortes por ano são relacionadas a doenças cardiovasculares em todo o mundo; dentre elas, doença isquêmica do coração e doenças cerebrovasculares (BEAGLEHOLE et al., 2001).

No Brasil, segundo pesquisas do Ministério da Saúde, as doenças cardiovasculares correspondem a 1/3 dos óbitos por causas conhecidas e quase $2 / 3$ dos gastos com atenção à saúde em 2002 (BARBOSA, 2003). 
Por meio das despesas com a aquisição de sal para consumo familiar, dados da POF 2002-2003 indicaram que o consumo de sal é, em média, estimado em 9,6g/pessoa/dia, ou seja, quase duas vezes maior que o recomendado, de $5 \mathrm{~g} / \mathrm{pessoa} / \mathrm{dia}$, o que corresponde a uma colher de chá rasa por pessoa (INSTITUTO BRASILEIRO DE GEOGRAFIA E ESTATÍSTICA, 2011).

O cloreto de sódio (sal de cozinha) é utilizado como tempero para realçar o sabor dos alimentos e, também, na conservação de alimentos industrializados. Além do cloreto de sódio, outros compostos químicos que contêm sódio em sua composição, como o glutamato de sódio, são muito usados pela indústria alimentar no processamento de diversos produtos (BRASIL, 2005).

Além disso, se computado o consumo de sódio fora de casa, esse valor fica ainda maior. O consumo de pizza, carnes processadas, salgadinhos industrializados, biscoito recheado e refrigerante foi relacionado ao consumo elevado de sódio e como marcador de consumo não saudável. O percentual de consumo fora do domicílio, em relação ao consumo total, foi de $53,2 \%$ para salgados fritos e assados e 56,5\% para salgadinhos industrializados (alimentos ricos em sódio), confirmando os grandes percentuais de inadequação da alimentação da população brasileira (INSTITUTO BRASILEIRO DE GEOGRAFIA E ESTATÍSTICA, 2011).

Para todo o país, a quantidade de sódio ofertado para consumo foi de 4,5 gramas por pessoa por dia (g/p/d), mais de duas vezes superior ao limite máximo de consumo de $2 \mathrm{~g} / \mathrm{p} / \mathrm{d}$ (SARNO, 2009). Restaurantes institucionais, que fornecem alimentação a trabalhadores, também se confrontam com o problema da oferta excessiva de sódio (SALAS, et al. 2009).

Nesse contexto, este trabalho se propõe a avaliar a quantidade de sódio extrínseco utilizado em preparações de uma unidade de alimentação e nutrição.

\section{METODOLOGIA}

Trata-se de um estudo de caráter observacional e transversal, realizado em uma unidade de alimentação de uma escola privada do município de São Paulo, no ano de 2012. A unidade estudada atende, aproximadamente, 60 clientes na hora do almoço - alunos, pais e professores. É um serviço terceirizado com refeição opcional.

O trabalho foi desenvolvido a partir do próprio interesse da empresa em melhorar seus processos produtivos.

A oferta total dos alimentos servidos foi obtida pesando-se todas as preparações e subtraindo-se as sobras, conforme descrito em Abreu, Spinelli e Pinto (2011). Os restos não foram analisados neste trabalho.

Para calcular a quantidade de sódio de cada preparação, foi pesada a quantidade de sal, de tempero industrializado para arroz e outros temperos ricos em sódio antes de serem utilizados no preparo. A quantidade de sódio referente à quantidade de cada tempero foi somada para chegar à quantidade de sódio por preparação. 
Com a quantidade de sódio estabelecida, o valor foi dividido entre o número de clientes do dia, criando uma média per capita de sódio por preparação.

Para chegar à quantidade de sódio por refeição, os per capitas de cada preparação foram somados e o número final foi considerado a média de oferta de sódio por cliente.

O resultado foi comparado com a recomendação de ingestão para a população brasileira, segundo o Guia Alimentar para a População Brasileira (BRASIL, 2005) e com a recomendação do Programa de Alimentação do Trabalhador - PAT (BRASIL, 2006), uma vez que parte dos consumidores são trabalhadores do colégio.

Os dados foram digitados e processados em planilha eletrônica e foram revisados quanto à consistência. Para as variáveis numéricas, os dados foram apresentados em formas de tabelas, e as médias foram calculadas.

Questão Ética: Para atender aos princípios éticos, o responsável pelo restaurante, ao ser convidado a participar da pesquisa, foi esclarecido quanto aos objetivos do trabalho e recebeu a garantia do anonimato e do retorno dos resultados encontrados.

\section{RESULTADOS}

Todas as preparações que utilizam sal e temperos com alto teor de sódio foram analisadas e descritas nas Tabelas 1,2 e 3. Não foram computadas saladas e outras preparações que não utilizavam temperos no preparo.

Observa-se, na Tabela 1, que o arroz, um dos principais alimentos que integram a alimentação brasileira, foi responsável pela maior contribuição de sódio de adição nesse dia.

Tabela 1 - Valores de sódio nos alimentos e total per capita (dia 1). São Paulo, 2012

\begin{tabular}{lrrrrrr}
\hline Preparação & $\begin{array}{c}\text { Consumo } \\
\text { Total (g) }\end{array}$ & Sal (g) & $\begin{array}{c}\text { Tempero } \\
\text { industrializado } \\
\text { para arroz } \mathbf{( g )}\end{array}$ & $\begin{array}{c}\text { Outros } \\
\text { temperos } \\
(\mathbf{g})\end{array}$ & $\begin{array}{c}\text { Sódio } \\
(\mathbf{m g})\end{array}$ & $\begin{array}{r}\text { Per Capita } \\
\text { (56 clientes) }\end{array}$ \\
\hline Arroz & 8625 & 27,5 & 27,5 & 0 & 19134,5 & 341,7 \\
Feijão & 3840 & 23,4 & 11,7 & 0 & 12703,9 & 226,8 \\
Cação & 3350 & 29,1 & 11,2 & 0 & 14774,0 & 263,8 \\
Vagem & 1215 & 25,9 & 0 & $207,2 \mathrm{~g}$ & 10992,0 & 196,3 \\
& & & & & \\
Molho sugo & 1310 & 7,6 & 7,6 & 0 & 5288,0 & 94,4 \\
\hline \multicolumn{2}{l}{ Total Na per capita } & & & & & $\mathbf{1 1 2 3 m g}$ \\
\hline
\end{tabular}

$\mathrm{Na}$ Tabela 2, é possível notar que, também nesse dia, o arroz foi o alimento que contribuiu com o maior teor de sódio. 
Tabela 2 - Valores de sódio nos alimentos e total per capita (dia 2). São Paulo, 2012

\begin{tabular}{lrrrrrr}
\hline Preparação & $\begin{array}{c}\text { Consumo } \\
\text { Total (g) }\end{array}$ & Sal (g) & $\begin{array}{c}\text { Tempero } \\
\text { industrializado } \\
\text { para arroz (g) }\end{array}$ & $\begin{array}{c}\text { Outros } \\
\text { temperos } \\
(\mathbf{g})\end{array}$ & $\begin{array}{c}\text { Sódio } \\
\text { (mg) }\end{array}$ & $\begin{array}{r}\text { Per Capita } \\
\text { (52 clientes) }\end{array}$ \\
\hline Arroz & 6615 & 22,6 & 22,6 & 0 & 15725,0 & 302,4 \\
Feijão & 2615 & 9,8 & 9,8 & 0 & 6818,8 & 131,2 \\
$\begin{array}{l}\text { Fricassê de } \\
\text { Frango }\end{array}$ & 8065 & 13,2 & 21,1 & $\begin{array}{r}528,3 \mathrm{mg} \\
\text { margarina }\end{array}$ & 13881,2 & 266,9 \\
Escarola & 710 & 0 & 6,8 & $\begin{array}{r}54,4 \mathrm{~g} \\
\text { bacon }\end{array}$ & 2237,2 & 43,0 \\
Cenoura & 1915 & 22,63 & 13,6 & $\begin{array}{r}362,2 \\
\text { margarina }\end{array}$ & 14542,0 & 279,6 \\
\hline
\end{tabular}

Total Na per capita $1023,1 \mathrm{mg}$

Nota-se, na Tabela 3 que, nesse dia, o principal responsável pelo teor de sódio da refeição foi o prato protéico.

Tabela 3 - Valores de sódio nos alimentos e total per capita (dia 3). São Paulo, 2012

\begin{tabular}{|c|c|c|c|c|c|c|}
\hline Preparação & $\begin{array}{l}\text { Consumo } \\
\text { Total (g) }\end{array}$ & Sal (g) & $\begin{array}{c}\text { Tempero } \\
\text { industrializado } \\
\text { para arroz }(\mathrm{g})\end{array}$ & $\begin{array}{c}\text { Outros } \\
\text { temperos } \\
(\mathrm{g})\end{array}$ & $\begin{array}{l}\text { Sódio } \\
(\mathrm{mg})\end{array}$ & $\begin{array}{c}\text { Per Capita } \\
\text { (39 clientes) }\end{array}$ \\
\hline Arroz & 2725 & 6,5 & 6,5 & 0 & 4522,7 & 116,0 \\
\hline Feijão & 3299 & 15,6 & 0 & $\begin{array}{r}155,7 \mathrm{~g} \\
\text { bacon }\end{array}$ & 6535,5 & 167,6 \\
\hline $\begin{array}{l}\text { Patela de } \\
\text { Porco }\end{array}$ & 2336 & 43,9 & 0 & 0 & 17121,0 & 439,0 \\
\hline Couve & 2060 & 14,5 & 14,5 & 0 & 10089,1 & 258,7 \\
\hline $\begin{array}{l}\text { Batata } \\
\text { dourada }\end{array}$ & 2680 & 17,1 & 0 & 0 & 6669,0 & 171,0 \\
\hline \multicolumn{3}{|c|}{ Total Na per capita } & & & & $1152,3 \mathrm{mg}$ \\
\hline
\end{tabular}

\section{DISCUSSÃO}

Foi encontrado um valor médio de consumo individual de refeição de $346 \mathrm{~g}$ por pessoa, valor menor que o encontrado em outros estudos em unidades de alimentação, como no de Salas et al. (2009) - 711g; no de Spinelli et al. (2012) - 530g; e no de Magnée (1996), em restaurantes self-service, de, aproximadamente, $420 \mathrm{~g}$. O per capita menor na UAN do presente estudo possivelmente seja decorrente do tipo de usuário, composto, em parte, por estudantes do ensino médio.

Embora os per capitas das refeições dos três dias sejam baixos, o valor médio per capita de sódio encontrado na refeição está acima das recomendações (BRASIL, 2006).

Observam-se, nas Tabelas 1, 2 e 3, que os porcionamentos de arroz variaram entre 70 e 154g, com um teor de sódio entre 116,0 e 341,7mg de sódio, e os de feijão de 50 a $85 \mathrm{~g}$ com valores de sódio entre 131,2 e 226,8mg. Na pesquisa de Spinelli, Kawashima e Egashira (2011), em restaurantes comerciais da região de Suzano - SP, foi encontrado um teor de sódio 
médio de $321,6 \mathrm{mg}$, em $100 \mathrm{~g}$ de arroz, e de $346,6 \mathrm{mg}$, em $100 \mathrm{~g}$ de feijão, pouco maiores que os do presente estudo.

Em estudo realizado em Salvador por Assis et al. (2002), 95,6\% dos adultos entrevistados revelaram consumir feijão, sendo que 65,7\% disseram fazê-lo de 4 a 7 vezes na semana. Os resultados, para o arroz, nesse mesmo estudo, revelaram que 94,0\% consumiam esse alimento, e, destes, 91,5\% faziam uso de 4 a 7 vezes por semana. Segundo estudo de Mattos e Martins (2000), no almoço, o consumo de arroz e feijão se mostram predominantes (referido por $97,5 \%$ e $91,2 \%$ das pessoas, respectivamente, confirmando serem esses os itens básicos do padrão alimentar brasileiro. Esses dados confirmam a importância no controle do uso de sal e de condimentos industrializados nesses alimentos, pois, embora o arroz e o feijão apresentem quantidades ínfimas de sódio em sua composição (TACO, 2006), foram responsáveis pela maior contribuição de sódio em dois dos três dias pesquisados.

Conforme pode ser observado nas três tabelas, o tempero industrializado para arroz é utilizado em diversas preparações, sendo responsável por $29 \%$ do sódio total de adição. Barbosa, Barros e Spinelli (2012) também se referem ao excesso de oferta de sódio em restaurante industrial atribuído à grande utilização de condimentos e alimentos industrializados.

Em países industrializados, cerca de $75 \%$ do sódio consumido origina-se de alimentos processados e de alimentos consumidos fora do domicílio. Nos Estados Unidos, várias preparações servidas em restaurantes contêm mais de $2,3 \mathrm{~g}$ de sódio, o que equivale ao limite superior tolerável da ingestão adotada pelo país (WORLD HEALTH ORGANIZATION, 2007).

Fica evidenciada a importância da priorização de técnicas culinárias mais adequadas, privilegiando o uso de temperos naturais, como cebola, alho e ervas aromáticas, uma vez que esses valores diários de sódio podem, com o tempo, representar um risco à saúde dos clientes da unidade.

Nas preparações de uso diário, como arroz e feijão, encontrou-se uma grande disparidade de valores de sódio, que pode ser atribuída à falta ou mesmo ao não uso de receituário padrão. O receituário padrão é um dos principais instrumentos de controle do restaurante, permitindo uma padronização da qualidade, uma vez que, atualmente, esta é exigida nos serviços, não admitindo empirismos e com benefício direto para a saúde do consumidor (ABREU; SPINELLI; PINTO, 2011).

Se as preparações de carne fossem padronizadas em porções de $100 \mathrm{~g}$, haveria uma variação de sódio de 232,1mg (correspondente ao "fricassé" de frango) a 731,6mg (correspondente à patela de porco), ou seja, é 24,2 a $76,2 \%$ do valor máximo de sódio, preconizado pelo PAT, para uma refeição (BRASIL, 2006). Spinelli, Kawashima e Egashira (2011) também referem à grande variação nos teores de sódio, nas preparações de carnes, atribuídas a condimentos industrializados e amaciantes para carnes.

Chama a atenção os valores de sódio fornecidos pelas hortaliças, tidos como "alimentos saudáveis" no consenso geral, foram responsáveis por 25,9\%, 31,5\% e 37,2\% do valor total 
de sódio nos dias 1, 2 e 3, respectivamente, como pode ser observado nas Tabelas 1, 2 e 3. Quando oferecidas na forma de salada, recebem a adição de condimentos extras. Com relação ao valor máximo preconizado pelo PAT, para uma única refeição, elas representam $30,3 \%, 33,6 \%$ e $44,8 \%$, respectivamente, embora o porcionamento tenha sido pequeno nos dias 1 e 2.

Além das preparações servidas, são dispostos sachês de 1 grama de sal na bancada de distribuição, sendo a sua utilização, livre para todos os clientes. Cada cliente, adulto ou adolescente, utiliza, em média, meio sachê por refeição. Cada meio sachê contém 195mg de sódio, o que eleva o per capita médio de sódio a 1294,5mg/refeição, ou seja, metade da necessidade diária, sem considerar as demais fontes de sódio provindas das bebidas consumidas durante o almoço. Esse resultado é coerente com os dados da POF 2002-2003, que estimam um consumo de sódio pela população brasileira maior que o recomendado (BRASIL, 2005).

O valor encontrado nessa pesquisa se assemelha ao que a literatura descreve como comum em unidades de alimentação. Salas et al. (2009) verificaram uma oferta diária de sódio correspondente ao dobro da recomendação do PAT para uma refeição, praticamente ultrapassando a recomendação diária de ingestão de sal da Organização Mundial da Saúde.

O grande consumo de sódio pode aumentar os riscos de doenças coronarianas e vasculares, porém há grande resistência quanto à mudança desse hábito pela população. Um aspecto a ser considerado, nesse processo de educação alimentar, é que, ao tentar diminuir esse consumo, pessoas habituadas a alimentos salgados tendem a considerar o sabor dos alimentos não tão satisfatórios. As células do paladar podem levar até 3 meses para se ajustar ao sabor menos intenso do sal e é importante que as pessoas saibam disso para persistirem no consumo de alimentos com menor teor de sal (BRASIL, 2005). Para Proença et al. (2005), a adaptação das papilas gustativas acontece tanto para a redução como para o aumento do teor de sal da alimentação e quem optar pela redução da quantidade de sal, na alimentação, não conseguirá ter o mesmo prazer consumindo os alimentos com a quantidade de sal usada anteriormente.

\section{CONCLUSÃO}

O consumo de sódio nessa unidade estudada é alto.

A variação das quantidades de sódio nos alimentos estudados e o uso excessivo de sal e de condimentos industrializados evidenciam a necessidade de criação e utilização de receituário técnico, padrão, para garantir uma refeição mais saudável ao consumidor.

É importante que se faça uma adequação como medida de prevenção de risco à saúde e com caráter educativo, já que se trata de refeição de unidade escolar.

Os serviços de alimentação têm, por dever, proteger a saúde de seus clientes e colaboradores, e, portanto, devem ser mais criteriosos com a quantidade de componentes prejudiciais à saúde utilizados em suas preparações, além de padronizarem as receitas, 
indicando as quantidades de sal a serem utilizadas.

\section{REFERÊNCIAS}

ABREU, E. S.; SPINELLI, M. G. N.; PINTO, A. M. S. Gestão de unidades de alimentação e nutrição: um modo de fazer. 4. ed. São Paulo: MEtha, 2011.

ASSIS, A. O. et al. Perfil alimentar e nutricional de adolescentes, adultos e idosos da cidade de Salvador: Relatório técnico. Salvador: Ministério as Saúde; Secretaria da Saúde do Estado da Bahia, 2002.

BARBOSA, J. Doenças não transmissíveis: tema relevante para a vigilância em saúde pública no Brasil. In: III Fórum global de prevenção e controle de doenças não transmissíveis, 3, 2003. Rio de Janeiro, [Anais...]. Rio de Janeiro, 2003.

BARBOSA, M. R. A. S.; BARROS, V. A. M. M.; SPINELLI, M. G. N. Teores de sódio em refeições servidas em unidade de alimentação e nutrição na cidade de São Paulo - SP. Nutrição em Pauta, v. 117, pp. 21-24, 2012.

BEAGLEHOLE, R. et al. Cardiovascular diseases: causes, surveillance and prevention. I International Journal of Epidemiology, v. 30, pp. S1-S4, Oct. 2001.

BRASIL. Ministério da Saúde. Secretaria de Atenção à Saúde. Coordenação Geral da política de Alimentação e Nutrição. Guia Alimentar para a População Brasileira: promovendo a alimentação saudável. Brasília: Ministério da Saúde, 2005. (Série A. Normas e Manuais Técnicos).

BRASIL. Ministério da Saúde. Secretaria de Atenção à Saúde. Coordenação Geral da política de Alimentação e Nutrição. Guia Alimentar para a População Brasileira: Promovendo a alimentação saudável. Brasília: Ministério da Saúde, 2008.

BRASIL. Ministério do Trabalho e Emprego. Portaria Interministerial n. 66, de 25 de agosto de 2006. Altera os parâmetros nutricionais do Programa de Alimentação do Trabalhador - PAT. Diário Oficial da União, 28 de agosto, 2006.

INSTITUTO BRASILEIRO DE GEOGRAFIA E ESTATÍSTICA. Pesquisa de orçamentos familiares 2008-2009: análise do consumo alimentar pessoal no Brasil. Rio de Janeiro: IBGE, 2011.

MAGNÉE, H. M. Manual do self-service. São Paulo: Varela, 1996.

MATTOS, L. L.; MARTINS, I. S. Fibras alimentares em população adulta. Revista de Saúde Pública, São Paulo, v. 34, n. 1, pp. 50-55, 2000.

PROENÇA, R. P. C. et al. Qualidade nutricional e sensorial na produção de refeições. Florianópolis: EdUFSC, 2005. 
SALAS, C. K. T. S. et al. Teores de sódio e lipídios em refeições almoço consumidas por trabalhadores de uma empresa do município de Suzano, SP. Revista de Nutrição, Campinas, v. 22, n. 3, pp. 331-339, jun. 2009.

SALAS, C. K. T. S.; SPINELLI, M. G. N. Avaliação do consumo de sódio e lipídios em almoço de uma Unidade de Alimentação e Nutrição. São Paulo: CRN3, 2007. (Prêmio Maria Lúcia Ferrari Cavalcanti).

SARNO, F. et al. Estimativa de consumo de sódio pela população brasileira, 2002-2003. Revista de Saúde Pública, São Paulo, v. 43, n. 2, abr. 2009.

SPINELLI, M. G. N.; KAWASHIMA, L. M.; EGASHIRA, E. M. Análise de sódio em preparações habitualmente consumidas em restaurantes self-service. Alimentos e Nutrição, Araraquara, v. 22, pp. 55-61, 2011.

SPINELLI, M. G. N. et al. Porcionamento de alimentos em unidades de alimentação e nutrição com distribuição self-service. Rev Nutrição Profissional, v. 34, pp. 29-38, 2012.

TACO. Tabela brasileira de composição de alimentos. 2. ed. Campinas: NEPA; UNICAMP, 2006.

WORLD HEALTH ORGANIZATION. Reducing salt intake in populations. Report of a WHO forum and technical meeting. Geneva: WHO, 2007. 